\title{
Exploring the expression of defence-related genes in Actinidia spp. after infection with Pseudomonas syringae pv. actinidiae and pv. actinidifoliorum: first steps
}

\author{
M. Nunes da Silva',2, J. Machado², G.M. Balestra ${ }^{3}$, A. Mazzaglia ${ }^{3}$, M.W. Vasconcelos ${ }^{2}$ and \\ S.M.P. Carvalho ${ }^{1}$ \\ ${ }^{1}$ GreenUPorto - Research Centre for Sustainable AgriFood Production \& DGAOT, Faculdade de Ciências da Universidade \\ do Porto, Vairão, Portugal \\ ${ }^{2}$ Universidade Católica Portuguesa, Centro de Biotecnologia e Química Fina (CBQF), Laboratório Associado, Escola \\ Superior de Biotecnologia, Porto, Portugal \\ ${ }^{3}$ Department of Agriculture and Forestry Science, Viterbo, Italy
}

\section{Summary}

Kiwifruit bacterial canker (KBC), caused by Pseudomonas syringae pv. actinidiae (PSA), is currently the most destructive disease of kiwifruit worldwide. Conversely, a closely related bacterial strain, $P$. syringae pv. actinidifoliorum (PFM), only causes necrotic spots and has not been associated with plant mortality. Moreover, there is some evidence on the higher susceptibility of the Actinidia chinensis var. deliciosa kiwifruit species to $\mathrm{KBC}$, compared with $A$. arguta, but the reasons behind it are still largely unknown. In this work, micropropagated plants of Actinidia chinensis var. deliciosa 'Hayward' and A. arguta var. arguta 'Ken's Red' were inoculated with PSA or with PFM $\left(10^{7}\right.$ CFUs $\left._{\mathrm{mL}^{-1}}\right)$. Disease development was monitored 1, 2 and 5 days post inoculation (dpi) through the determination colony forming units (CFUs) and the expression analysis of six plant defence-related genes (APX, CAT, SOD, LOX1, SAM and TLP1). At $5 \mathrm{dpi}$, CFUs in plant tissues inoculated with PSA and PFM were, respectively, 17.4-fold and 2.8-fold higher in A. chinensis compared with A. arguta. Expression of antioxidant enzyme-related genes was very distinct between the two kiwifruit species: SOD expression was drastically increased in $A$. chinensis (up to 2.1fold, 5 dpi), whereas in A. arguta CAT was the most upregulated gene (up to 1.7-fold, $2 \mathrm{dpi}$ ). LOX1, involved in jasmonic acid biosynthesis, was upregulated in both species, however reaching the highest values at 2 dpi in $A$. chinensis (2.2-fold) and 1 dpi in $A$. arguta (1.9-fold). It is concluded that $A$. arguta is much more tolerant to PSA than $A$. chinensis and that the molecular mechanisms between the two kiwifruit species involve specific defence pathways being triggered at distinct moments after plant infection.

Keywords

antioxidant enzymes, bacterial canker, kiwifruit, PFM, PSA, susceptibility

\section{Introduction}

Kiwifruit bacterial canker $(\mathrm{KBC})$ is currently the most destructive disease of kiwifruit worldwide. It is caused by Pseudomonas syringae pv. actinidiae (PSA) and it affects all

\section{Significance of this study}

What is already known on this subject?

- A. chinensis seems to be more susceptible to kiwifruit bacterial canker (KBC) than A. arguta; however, most of the available information is based on empirical observations. Moreover, A. chinensis infection with PSA enhances the expression of genes encoding antioxidant enzymes, but there is no information on other molecular pathways that may be involved in plant defence mechanisms, neither on how different Actinidia spp. respond to infection with $P$. syringae strains with distinct virulence.

What are the new findings?

- This is the first report where the higher susceptibility of $A$. chinensis to KBC, compared with A. arguta, is experimentally confirmed. The density of the highly virulent PSA strain rapidly increased in A. chinensis tissues, whereas in A. arguta bacterial colonization occurred later on and with a much lower magnitude. Contrastingly, the density of the low virulent PFM strain was consistently lower in both plant species. The catalase-encoding gene was the most upregulated in $A$. arguta plants 2 dpi, whereas in $A$. chinensis the expression of the superoxide dismutase-encoding gene was highly increased $5 \mathrm{dpi}$.

What is the expected impact on horticulture?

- Due to the economic importance of kiwifruit production in the world, it is imperative to evaluate the susceptibility of different cultivars and understand their tolerance mechanisms. In this work, the molecular mechanisms triggered after infection with PSA and PFM proved to be distinct between $A$. chinensis and $A$. arguta. These results will contribute to the identification of molecular markers for the precocious detection of the disease, and in breeding programmes for the development of tolerant cultivars.

kiwifruit species, including the green fleshed kiwifruit $A c$ tinidia chinensis var. deliciosa and the kiwi berry A. arguta var. arguta. A. chinensis orchards are, in general, more affected than $A$. arguta (Vanneste et al., 2014). Nevertheless, 
the reasons behind this differential susceptibility remain unknown.

After PSA infection through stomata, lenticels and wounds, disease symptoms range from leaf spotting, shoot wilting, twig dieback, blossom necrosis and lenticels reddening. Systemic migration from leaves to shoots through xylem vessels induces extensive canker formation and further bacterial dispersal into the environment through the release of exudates (Ferrante et al., 2012). Interestingly, the closely related strain $P$. syringae pv. actinidifoliorum (PFM) is able to infect kiwifruit plants but does not cause systemic infection or plant death (Vanneste et al., 2013; Cunty et al., 2014).

It is presumed that during the early colonization stages plants are able to restrain their endophytic bacterial populations through the activation of basal defence mechanisms, such as increased synthesis of oxidative stress-related proteins (Petriccione et al., 2013). In fact, one of the earliest cellular responses succeeding an effective pathogen recognition by the host plant is the production of reactive oxygen species (ROS) through the consumption of oxygen in a cascade of reactions called oxidative burst. During this process, superoxide dismutase (SOD), ascorbate peroxidase (APX), and catalase (CAT) play very important roles in ROS detoxification, protecting plant cells from oxidative burst induced by pathogen invasion (Mittler et al., 2004). While SOD catalyzes the dismutation of superoxide $\left(\mathrm{O}^{2-}\right)$ to hydrogen peroxide $\left(\mathrm{H}_{2} \mathrm{O}_{2}\right)$, APX reduces $\mathrm{H}_{2} \mathrm{O}_{2}$ to water $\left(\mathrm{H}_{2} \mathrm{O}\right)$ by utilizing ascorbate as an electron donor and CAT dismutates $\mathrm{H}_{2} \mathrm{O}_{2}$ to oxygen $\left(\mathrm{O}_{2}\right)$ and water. In a recent study it was shown that the expression of genes encoding antioxidant enzymes, such as $S O D, A P X$, and $C A T$, increased after A. chinensis inoculation with PSA from as early as 1 day post inoculation (Petriccione et al., 2015). Nevertheless, information on other molecular pathways that may be involved in plant defence mechanisms and on how different Actinidia spp. respond to infection with $P$. syringae strains with distinct virulence are still very scarce.

The aim of this work was to evaluate how PSA (highly virulent) and PFM (less virulent) bacterial populations perform in A. chinensis var. deliciosa 'Hayward' (more susceptible) and A. arguta var. arguta 'Ken's Red' (more tolerant) plant tissues after artificial infection, and how these species with reported distinct susceptibility to $\mathrm{KBC}$ respond to the infection in terms of the expression of defence-related genes.

\section{Materials and methods}

\section{Plant maintenance}

Micropropagated A. chinensis var. deliciosa 'Hayward' and A. arguta var. arguta 'Ken's Red' plants were purchased from QualityPlant, Investigação e Produção em Biotecnologia Vegetal, Lda. (Castelo Branco, Portugal). A modified Murashige and Skoog (MS) agarized medium was used for plant maintenance during the trial period and consisted in sucrose (30 $\left.\mathrm{g} \mathrm{L}^{-1}\right)$, myo-inositol (100 $\left.\mathrm{mg} \mathrm{L}^{-1}\right)$, thiamine- $\mathrm{HCl}$ $\left(1 \mathrm{mg} \mathrm{L}^{-1}\right)$, nicotinic acid $\left(1 \mathrm{mg} \mathrm{L}^{-1}\right)$, pyridoxine $\left(1 \mathrm{mg} \mathrm{L}^{-1}\right)$, glycine ( $\left.1 \mathrm{mg} \mathrm{L}^{-1}\right)$ and benzylaminopurine $\left(0.5 \mathrm{mg} \mathrm{L}^{-1}\right)$, adjusted to $\mathrm{pH} 5.7$ with $\mathrm{KOH}$. Plants were kept in sets of three plants per OS140 box container (Duchefa Biochemie B.V., Haarlem, The Netherlands) in a climate chamber (Aralab Fitoclima 5000EH, Aralab, Rio de Mouro, Portugal) with 16-h day photoperiod with $200 \mu \mathrm{mol} \mathrm{s} \mathrm{s}^{-1} \mathrm{~m}^{-2}$ of photosynthetic photon flux density at plant level. Temperatures were set to $22^{\circ} \mathrm{C}$ during the light period and to $19^{\circ} \mathrm{C}$ during the dark period and relative humidity was maintained at $80 \%$.

\section{Bacterial suspension preparation and plant inoculation}

A virulent PSA strain (CFBP7286, isolated in 2008 in Italy from $A$. chinensis) and a less virulent PFM strain (ICMP18804, isolated in 2010 in New Zealand from A. chinensis) were grown for $48 \mathrm{~h}$ on nutrient agar with $5 \%$ sucrose (NSA) at $27^{\circ} \mathrm{C}$ in the dark. In the day of inoculation, a fresh $1-2 \times 10^{7} \mathrm{CFUs} \mathrm{mL}^{-1}$ inoculum was prepared in sterile Ringer's solution $\left(\mathrm{NaCl} 0.72 \%, \mathrm{CaCl}_{2} 0.017 \%\right.$ and $\mathrm{KCl} 0.037 \%$, $\mathrm{pH}$ 7.4). Plants were inoculated with bacterial suspension by gently rubbing a sterile swab impregnated with one of the solutions on the abaxial surface of each leaf. For each kiwifruit species (A. chinensis and A. arguta) and P. syringae strain (PSA and PFM), 27 plants were inoculated (i.e., nine containers with three plants each). Control non-inoculated plants were treated with Ringer's solution alone.

\section{Plant sampling}

One, 2 and 5 days post inoculation (dpi), three containers of each kiwifruit species and $P$. syringae strain (with three plants per container) were randomly selected for plant sampling. Plants were removed from the culturing medium and the tip (ca. $0.5 \mathrm{~cm}$ length) of every leaf was cut with sterile scissors and placed in a sterile container for CFUs determination. The remaining plant was flash-frozen in liquid nitrogen and stored at $-80^{\circ} \mathrm{C}$ for gene expression analysis. Each biological replicate was obtained by pooling the three plants per container, and three independent biological replicates were analysed per treatment at each time-point.

\section{CFUs determination in plant tissues}

Estimation of bacterial colony forming units (CFUs) was performed using an adapted method from Cellini et al. (2014). Samples were surface sterilised by washing in $70 \%$ ethanol for $1 \mathrm{~min}$, followed by a 1-min treatment with $1 \%$ sodium hypochlorite, after which they were rinsed twice in sterile water for $1 \mathrm{~min}$. Plant samples were then homogenised in $10 \mathrm{~mL}$ Ringer's solution. This extract was sequentially diluted ten-fold up to $10^{-5}$, and three replicates of $100 \mu \mathrm{L}$ from each ten-fold dilution were plated on NSA medium. After plate incubation at $27^{\circ} \mathrm{C}$ for $48 \mathrm{~h}$ in the dark, the number of colonies in each plate were counted and CFUs estimated.

\section{Defence-related gene expression analysis}

Plant RNA was extracted according to an adapted protocol from Cellini et al. (2014). After tissue homogenization with liquid nitrogen, $1 \mathrm{~mL}$ of warm $\left(70^{\circ} \mathrm{C}\right)$ extraction buffer (100 mM Tris- $\mathrm{HCl}, \mathrm{pH}$ 8.0, cetyltrimethylammonium bromide $4 \% \mathrm{w} / \mathrm{v}$, polyvinylpyrrolidone $\mathrm{K} 404 \% \mathrm{w} / \mathrm{v}, 30 \mathrm{mM}$ ethylenediamine tetraacetic, $2.0 \mathrm{M} \mathrm{NaCl}$, spermidine $0.1 \%$ $\mathrm{w} / \mathrm{v}, \beta$-mercaptoethanol $2 \% \mathrm{v} / \mathrm{v}$ ) was added to ca. $100 \mathrm{mg}$ of sample. Samples were mixed vigorously and incubated at $65^{\circ} \mathrm{C}$ for $10 \mathrm{~min}$. Subsequently, $1 \mathrm{~mL}$ of chloroform-isoamyl alcohol (24:1, v/v) was added, and samples were centrifuged for $15 \mathrm{~min}$ at $15,000 \mathrm{~g}$. The upper phase was collected to a new tube and combined with $250 \mu \mathrm{L}$ of $12 \mathrm{M}$ $\mathrm{LiCl}$ by gentle pipetting. Samples were incubated overnight at $-20^{\circ} \mathrm{C}$, after which they were centrifuged at $15,000 \mathrm{~g}$ for $35 \mathrm{~min}$ at $4^{\circ} \mathrm{C}$. The supernatant was discarded and the pellet was washed in cold $70 \%$ ethanol, dried, and resuspend- 
TABLE 1. Primer sequences used for transcriptional analysis by qRT-PCR.

\begin{tabular}{llll}
\hline \multirow{2}{*}{$\begin{array}{l}\text { Gene } \\
\text { (Accession number) }\end{array}$} & \multicolumn{2}{c}{ Primer sequence (5'-3') } & Reference \\
\cline { 2 - 3 } ACT (FG440519) & CCAAGGCCAACAGAGAGAAG & GACGGAGGATAGCATGAGGA & Ledger et al. (2010) \\
PP2A (FG522516) & GCAGCACATAATTCCACAGG & TTTCTGAGCCCATAACAGGAG & Nardozza et al. (2013) \\
APX (FG408540) & GGAGCCGATCAAGGAACAGT & AACGGAATATCAGGGCCTCC & Petriccione et al. (2015) \\
CAT (FG470670) & GCTTGGACCCAACTATCTGC & TTGACCTCCTCATCCCTGTG & \\
SOD (FG471220) & CACAAGAAGCACCACCAGAC & TCTGCAATTTGACGACGGTG & This study \\
LOX (DQ497792) & GTTAGAGGGGTGGTGACTCT & CTTTAGCACTGCTTGGTTGC & \\
SAM (U17240) & GAATAGTACTTGCCCCTGGC & TACAAATCGACCAGAGGGGT & \\
TLP1 (JX905282) & CAACCCCCTAACACACTAGC & ATTTCCGGAGTTGCAACAGT & \\
\hline
\end{tabular}

ed in $40 \mu \mathrm{L}$ sterile DEPC water. Single-stranded cDNA was synthesized using iScript cDNA Synthesis Kit (Bio-Rad, California, USA) according to the manufacturer's instructions in a Doppio Thermal Cycler (VWR, Oud-Heverlee, Belgium).

Primers for LOX1, SAM and TLP1 were designed using Primer3 (Frodo.wi.mit.edu) for an expected PCR product of 100-200 bp and primer annealing temperatures between 56 and $58^{\circ} \mathrm{C}$, whereas primer sequences for $A P X, C A T$ and $S O D$ were obtained from Petriccione et al. (2015) (Table 1). Reverse transcription polymerase chain reactions (qRTPCR) were performed on a StepOne ${ }^{T M}$ Real-Time PCR System (Applied Biosystems, California, USA) with the following reaction conditions: $2 \mathrm{~min}$ at $50^{\circ} \mathrm{C}, 2 \mathrm{~min}$ at $95^{\circ} \mathrm{C}$ and 40 cycles with: $15 \mathrm{~s}$ at $95^{\circ} \mathrm{C}, 15 \mathrm{~s}$ at each primer pair optimal annealing temperature (Table 1 ) and $1 \mathrm{~s}$ at $72^{\circ} \mathrm{C}$. Amplifications were carried out using a final volume of $20 \mu \mathrm{L}$ which consisted of $1 \mu \mathrm{L}$ of the specific primers at $6 \mu \mathrm{M}$, $10 \mu \mathrm{L}$ of $2 \times$ iQ SYBR ${ }^{\circledR}$ Green Supermix (Bio-Rad, California, USA) and $8 \mu \mathrm{L}$ of a 1:100 dilution of the template cDNA. Melt curve profiles were analysed for each tested gene. The comparative CT method ( $\Delta \Delta \mathrm{CT}$, Livak and Schmittgen, 2001) was used for the relative quantification of gene expression values using actin $(A C T)$ and protein phosphatase $2 \mathrm{~A}$ (PP2A) genes as control transcript (Petriccione et al., 2015) and the plants inoculated with Ringer's solution (controls) as the reference sample. For each sample and target gene two technical replicates were analysed.

\section{Statistical analysis}

Data were analysed with GraphPad Prism version 6.0 (GraphPad Software, Inc., California, USA). Differences between treatments were tested with ANOVA corrected for multiple comparisons using the Tukey method $(p<0.05)$.

\section{Results and discussion}

After PSA infection through natural openings and wounds, bacteria start to migrate within the vascular system inducing a wide variety of disease symptoms that range from leaf spotting to cankers formation (Ferrante et al., 2012). Contrarily, PFM has lower virulence in kiwifruit plants since it causes a non-systemic infection, only being associated with leaf symptoms (Cunty et al., 2014). In this work, CFUs determination showed rapid PSA colonization in kiwifruit plant tissues, especially in A. chinensis. In fact, at $2 \mathrm{dpi} A$. chinensis plants had ca. $235 \pm 23 \times 10^{3}$ CFUs $\mathrm{mL}^{-1}$ for the highly virulent Italian PSA strain (Figure 1), whereas in A. arguta PSA CFUs values remained ca. 6.7-fold lower $\left(35 \pm 12 \times 10^{3} \mathrm{CFUs} \mathrm{mL}^{-1}\right)$. At $5 \mathrm{dpi}, A$. chinensis had 17.4 times higher PSA CFUs count than $A$. arguta, which presented only ca. $75 \pm 7 \times 10^{3} \mathrm{CFUs}_{\mathrm{mL}^{-1}}$.
Rapid PSA population increase in artificially inoculated A. chinensis plants was already observed during the first days after inoculation by Petriccione et al. (2014). Here we show for the first time that the less virulent strain PFM seems to take a longer period of time to infect plant tissues, as this strain was only detected $5 \mathrm{dpi}$, and in much lower numbers compared to PSA (representing only 1.5\% and 9.3\% CFUs for $A$. chinensis and A. arguta respectively, when comparing to PSA). Similarly to PSA, CFUs following PFM infection were higher in $A$. chinensis than in A. arguta at $5 \mathrm{dpi}$ (ca. 2.8-fold, Figure 1). Although A. arguta susceptibility to PSA was already demonstrated in 1989 through artificial inoculation of nursery plants, it was only more recently that PSA was identified in A. arguta field plants for the first time (Vanneste et al., 2014). A. chinensis, on the other hand, is more commonly associated with PSA infection, frequently accompanied by extensive infection symptoms, such as cane dieback, cankers formation, release of bacterial exudates, and fruit production losses (Balestra et al., 2009). Results presented here corroborate that $A$. chinensis is more susceptible to PSA infection than A. arguta. Moreover, PSA and PFM establishment in plant tissues does not occur immediately after inoculum application and the rate of bacterial colonization is highly dependent on plant species and bacterial strain. It is hypothesised that leaf trichomes play

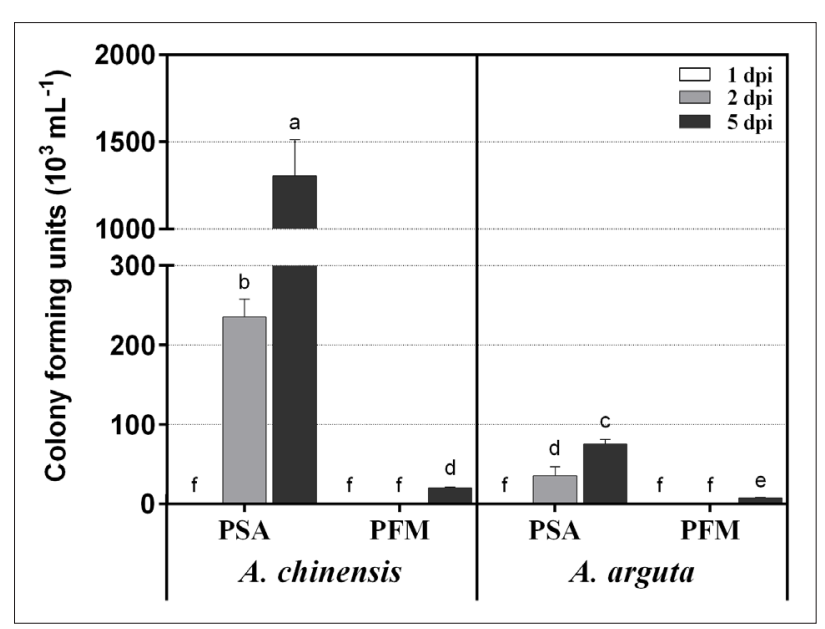

FIGURE 1. Number of colony forming units estimated in plant tissues from A. chinensis var. deliciosa 'Hayward' and A. arguta var. arguta 'Ken's Red' at 1, 2 and 5 days post inoculation (dpi) with $P$. syringae pv. actinidiae (PSA) or P. syringae pv. actinidifoliorum (PFM). Each bar represents the mean of three biological replicates \pm SE. Columns with the same letter are not significantly different at $p<0.05$. 
a very important role as an entry route for PSA to the host tissues (Spinelli et al., 2011). These authors reported that A. chinensis var. chinensis had higher trichome density in comparison to A. chinensis var. deliciosa, which could possibly contribute to the higher susceptibility of var. deliciosa, once they could provide a more favourable environment for bacterial growth. As A. chinensis leaves have higher trichome density than A. arguta, PSA and PFM could strive better in the first species than in the latter, therefore explaining the higher CFUs estimation in A. chinensis, regardless of bacterial strain or time-point. In addition, the fact that PSA was able to colonize plant tissues from an earlier stage after inoculation and to a greater magnitude, comparing with PFM, may be due to its systemic activity. In fact, as PSA can penetrate and migrate within plant leaf veins to the shoot (Petriccione et al., 2013), it can then multiply more rapidly, thus arising to greater numbers than PFM.

It has been recently shown that the expression of antioxidant enzymes-encoding genes play an important role in $A$. chinensis defence against PSA infection (Petriccione et al., 2015). These authors found that the upregulation of $S O D, A P X$ and $C A T$ genes was influenced not only by the bacterial concentration used for plant inoculation, but also by the time-point analysed. $S O D$ was shown to be significantly up-regulated in A. chinensis plants $7 \mathrm{dpi}$ after artificial inoculation with PSA (Petriccione et al., 2015). In the present study, the expression of these antioxidant enzymes-encoding genes were investigated not only in $A$. chinensis but also in $A$. arguta, following inoculation with two closely related but pathogenically distinct $P$. syringae. Here, SOD transcriptional levels significantly increased by 2.1 - and 1.7 -fold from 1 to 5 dpi after $A$. chinensis inoculation with PSA and PFM, respectively. Interestingly, in $A$. arguta inoculated plants no variation in $S O D$ transcriptional levels was observed over time, but there was a significant down-regulation of this gene in inoculated plants compared with non-infected plants (as the relative fold change was lower than 1), regardless of the bacterial strain (Figure 2). As SOD catalyzes the dismutation of $\mathrm{O}^{2-}$ to $\mathrm{H}_{2} \mathrm{O}_{2}$, the gradual increase in $S O D$ transcription following the inoculation of $A$. chinensis may be due to the cumulative concentration of $\mathrm{H}_{2} \mathrm{O}_{2}$ in plant cells in response to the increased PSA population (Figure 2).

Contrarily to $S O D, C A T$ transcriptional profile in A. chinensis plants did not show significant alterations during the experimental period, which seems to be in accordance with the results from Petriccione et al. (2015), where significant up-regulation of $C A T$ expression in PSA-inoculated plants was only observed $7 \mathrm{dpi}$. In $A$. arguta, on the other hand, CAT was up-regulated by 1.6- and 1.7-fold from 1 to $2 \mathrm{dpi}$, after inoculation with PSA and PFM, respectively. Perhaps in this kiwifruit species CAT enzyme plays a more determinant role in the prevention of oxidative stress, whereas in A. chinensis SOD seems to have a more active role (Figure 2).

Petriccione et al. (2015) reported a slight up-regulation in APX transcriptional levels 1 and $4 \mathrm{dpi}$ in A. chinensis var. deliciosa 'Hayward' following inoculation with PSA. In contrast, in the present study PSA inoculation induced a 0.4-fold decrease in APX expression in A. chinensis from 1 to $5 \mathrm{dpi}$, whereas in $A$. arguta it remained constant during the experimental period, being ca. 0.25 -fold higher than the non-inoculated plants (Figure 2). It was also found that 1 to $2 \mathrm{dpi}$ with the less virulent strain (PFM) A. chinensis responded with $A P X$ overexpression (ca. 1.3-fold), whereas $A$. arguta was not significantly affected. Moreover, when $A$. arguta plants were inoculated with PFM, $A P X$ was down-regulated by up to 0.4 -fold, compared with PSA, in all time-points. Therefore, it is clear that plant infection with bacterial pathovars with distinct virulence induces differentiated responses regarding the expression of genes related to the antioxidant pathways.

LOX1 encodes a lipoxygenase involved in the synthesis of oxygenated fatty acids, including jasmonic acid and aldehydes, which play important functions in plant defence against pathogens and herbivores (Kolomiets et al., 2000). Compared with control plants, the relative expression of this gene was 2.2- and 1.8-fold higher in PSA-inoculated A. chinensis and A. arguta plants $2 \mathrm{dpi}$ and $1 \mathrm{dpi}$, respectively, being drastically reduced afterwards in both species (Figure 2). Interestingly, lipoxygenases convert $\alpha$-linolenic acid into 13-hydroperoxyoctadecatrienoic acid in the jasmonic acid biosynthesis, a known antagonist to Actinidia spp. defence mechanisms. In fact, previous studies showed that exogenous application of methyl jasmonate, a synthetic analogue of jasmonic acid, increases $A$. chinensis and $A$. chinensis plants disease index after inoculation with PSA (Reglinski et al., 2013), and seems to impair salicylic acid pathway and increase Actinidia spp. susceptibility to PSA (Cellini et al., 2014). What is more, several $P$. syringae strains have the ability to produce the toxin coronatine, which disrupts plant defences by impairing plant ethylene and jasmonic acid pathways (Zheng et al., 2012). LOX1 increased activity was already reported in $A$. chinensis PSA-inoculated plants, and is regarded as a strategy used by the pathogen to antagonize salicylic acid responses through the enhancement of the jasmonic acid pathway, or as a consequence of the activation of other metabolic pathways, such as ethylene (Cellini et al., 2014).

S-adenosylmethionine synthetase encoding gene, SAM, is a precursor of ethylene biosynthesis and was up-regulated in A. chinensis by 1.4-fold after inoculation with PSA from 1 to $2 \mathrm{dpi}$ and from 1 to $5 \mathrm{dpi}$ in PFM-inoculated plants (Figure 2). In A. arguta, SAM was up-regulated by 2.2 -fold 1 day after PSA inoculation, compared with control plants. Exogenous application of ethylene was found to enhance disease index after PSA inoculation, probably because it antagonises salicylic acid pathway (Cellini et al., 2014). Contrarily, exogenous application of an ethylene receptor blocker, 1-MCP, decreased disease severity, thus supporting the evidence that ethylene may enhance the noxious effects of the pathogen, as was already demonstrated in other plant-pathogen systems (Weingart et al., 2001). In this report, genes involved in the methyl jasmonate and ethylene biosynthesis pathways (LOX1 and SAM) seemed to be triggered latter in $A$. chinensis, compared to A. arguta, since in the latter cultivar the upregulation of these genes, compared with control plants, peaked at 1 dpi. Moreover, the fact that $A$. arguta PSA-inoculated plants showed higher LOX1 and SAM transcriptional levels than PFM at $1 \mathrm{dpi}$ (1.6- and 2.0-fold, respectively) and in 'Hayward' at $2 \mathrm{dpi}$ (1.6-fold for both genes) clearly demonstrates that plants' defence mechanisms are highly influenced by the bacterial strain. Early deactivation of these pathways in A. arguta can be a coping mechanism against invasion by pathogenic bacteria, and underpin its higher tolerance to PSA and PFM.

TLP1 encodes a thaumatin-like protein, which belongs to the family of pathogenesis-related (PR) proteins, and is involved in acquired resistance and stress response in plants. These PR proteins were already shown to be up-regulated in PSA-infected $A$. chinensis plants as soon as $1 \mathrm{dpi}$ (Cellini et al., 2014). In this report, TLP1 was significantly 
up-regulated in PSA-inoculated plants from 1 to 5 dpi in A. chinensis and from 1 to $2 \mathrm{dpi}$ in A. arguta by ca. 1.4 -fold (Figure 2). Several studies have already demonstrated that TLPs have strong antifungal properties, probably due to its ability to inhibit the activities of several fungal enzymes, such as $\beta$-glucanase, xylanase, $\alpha$-amylase and trypsin, and also due to its ability to rupture fungal membrane by pore formation (Misra et al., 2016). The fact that this gene was up-regulated at earlier stages of the infection in A. arguta may reinforce its higher tolerance to PSA, compared with A. chinensis, or perhaps in A. chinensis other PR-proteins play a more preponderant role, at least in the time-points selected in this study.

\section{Conclusion}

A. chinensis var. deliciosa 'Hayward' proved to be much more susceptible to PSA infection than A. arguta var. arguta 'Ken's Red'. Moreover, PSA tolerance seems to be a result

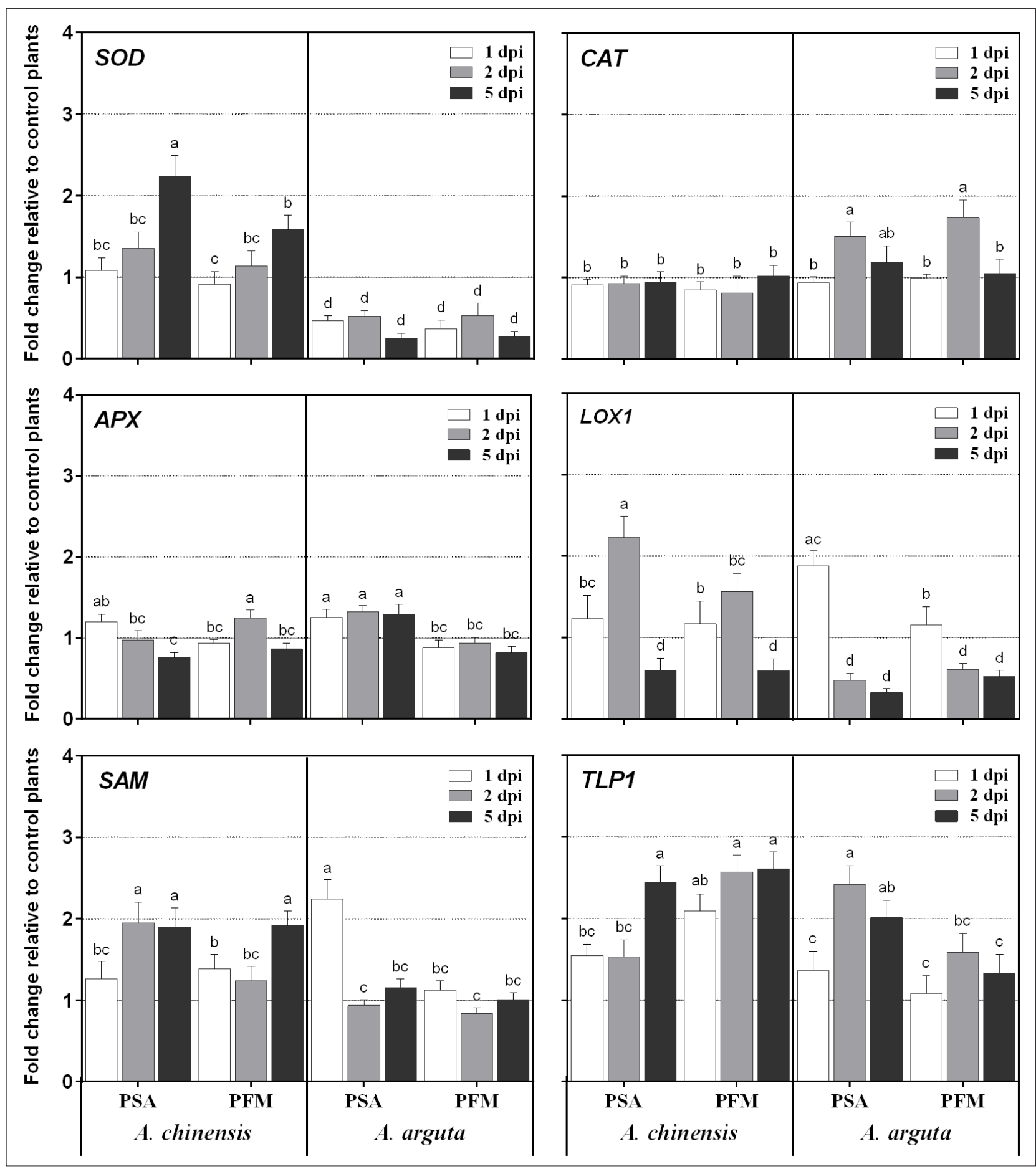

FIGURE 2. Gene expression analysis of SOD, CAT, APX, LOX1, SAM and TLP1 in A. chinensis var. deliciosa 'Hayward' and $A$. arguta var. arguta 'Ken's Red' at 1, 2 and 5 days post inoculation (dpi) with $P$. syringae pv. actinidiae (PSA) or $P$. syringae pv. actinidifoliorum (PFM), compared with non-inoculated control plants (1-fold change represents no relative change in gene expression). Each bar represents the mean of three biological replicates \pm SE relative to the housekeeping genes $A C T$ and $P P 2 A$ and to control plants. Columns with the same letter are not significantly different at $p<0.05$. 
of several plant resistance mechanisms acting together against the pathogen, as several defence-related genes were triggered at distinct moments after plant infection. Whereas $S O D$ expression was drastically increased in A. chinensis, in $A$. arguta CAT was the most upregulated antioxidant enzyme-encoding gene. Moreover, LOX1 and SAM, involved in jasmonic acid and ethylene biosynthesis, respectively, were upregulated $2 \mathrm{dpi}$ in $A$. chinensis and already at $1 \mathrm{dpi}$ in A. arguta and, more importantly, their transcriptional levels were higher in PSA-inoculated plants, compared with PFM. PSA may induce the upregulation of these pathways as part of its infection mechanism, impairing plant defence through negative feedback of the salicylic acid pathway.

Although these findings provide key evidence on the species-specific relation between Actinidia plants and $P$. syringae strains with different virulence, additional studies are needed to fully understand the molecular and metabolomic pathways involved in kiwifruit plants defence against PSA and PFM. Additionally, these results derive from in vitro grown Actinidia spp. plants and their reproducibility in fully grown field plants still needs to be confirmed. Nevertheless, the results presented here have the potential to contribute to the identification of molecular markers for the precocious detection of the disease and to the development of tolerant cultivars through breeding programmes.

\section{Acknowledgments}

The authors would like to thank Fundação para a Ciência e a Tecnologia (FCT) for funding (projects: PTDC/AGRPRO/6156/2014 and UID/Multi/50016/2013) and for MNS Ph.D. scholarship (SFRH/BD/99853/2014).

\section{References}

Balestra, G.M., Mazzaglia, A., Quattrucci, A., Renzi, M., and Rossetti, A. (2009). Current status of bacterial canker spread on kiwifruit in Italy. Australas. Plant Dis. Notes 4, 34-36.

Cellini, A., Fiorentini, L., Buriani, G., Yu, J., Donati, I., Cornish, D.A., Novak, B., Costa, G., Vanneste, J.L., and Spinelli, F. (2014). Elicitors of the salicylic acid pathway reduce incidence of bacterial canker of kiwifruit caused by Pseudomonas syringae pv. actinidae. Ann. Appl. Biol. 165, 441-453.

Cunty, A., Poliakoff, F., Rivoal, C., Cesbron, S., Fischer-Le Saux, M., Lemaire, C., Jacques, M.A., Manceau, C., and Vanneste, J.L. (2014). Characterisation of Pseudomonas syringae pv. actinidiae (Psa) isolated from France and assignment of Psa biovar 4 to a de novo pathovar: Pseudomonas syringae pv. actinidifoliorum pv. nov. Plant. Pathol. 64, 582-596. https://doi.org/10.1111/ppa.12297.

Ferrante, P., Fiorillo, E., Marcelletti, S., Marocchi, F., Mastroleo, M., Simeoni, S., and Scortichini, M. (2012). The importance of the main colonization and penetration sites of Pseudomonas syringae pv. actinidiae and prevailing weather conditions in the development of epidemics in yellow kiwifruit, recently observed in central Italy. J. Plant Pathol. 94, 455-461.

Kolomiets, M.V., Chen, H., Gladon, R.J., Braun, E.J., and Hannapel, D.J. (2000). A leaf lipoxygenase of potato induced specifically by pathogen infection. Plant Physiol. 124, 1121-1130. https://doi. org/10.1104/pp.124.3.1121.

Ledger, S.E., Janssen, B.J., Karunairetnam, S., Wang, T., and Snowden, K.C. (2010). Modified CAROTENOID CLEAVAGE DIOXYGENASE8 expression correlates with altered branching in kiwifruit (Actinidia chinensis). New Phytol. 188, 803-813. https://doi.org/10.1111/ j.1469-8137.2010.03394.x
Livak, K.J., and Schmittgen, T.D. (2001). Analysis of relative gene expression data using real-time quantitative PCR and the 2(-Delta Delta C(T)) Method. Meth. 25, 402-408. https://doi.org/10.1006/ meth.2001.1262.

Misra, R.C., Sandeep, Kamthan, M., Kumar, S., and Ghosh, S. (2016). A thaumatin-like protein of Ocimum basilicum confers tolerance to fungal pathogen and abiotic stress in transgenic Arabidopsis. Sci. Rep. 6, 25340. https://doi.org/10.1038/srep25340.

Mittler, R., Vanderauwera, S., Gollery, M., and Van Breusegem, F. (2004). The reactive oxygen gene network in plants. Trends Plant Sci. 9, 490-498. https://doi.org/10.1016/j.tplants.2004.08.009.

Nardozza, S., Boldingh, H.L., Osorio, S., Höhne, M., Wohlers, M., Gleave, A.P., MacRae, E.A., Richardson, A.C., Atkinson, R.G., Sulpice, R., Fernie, A.R., and Clearwater, M.J. (2013). Metabolic analysis of kiwifruit (Actinidia deliciosa) berries from extreme genotypes reveals hallmarks for fruit starch metabolism. J. Exp. Bot. 64, 50495063. https://doi.org/10.1093/jxb/ert293.

Petriccione, M., Di Cecco, I., Arena, S., Scaloni, A., and Scortichini, M. (2013). Proteomic changes in Actinidia chinensis shoot during systemic infection with a pandemic Pseudomonas syringae pv. actinidiae strain. J. Proteom. 78, 461-476. https://doi. org/10.1016/j.jprot.2012.10.014.

Petriccione, M., Salzano, A.M., Di Cecco, I., Scaloni, A., and Scortichini, M. (2014). Proteomic analysis of the Actinidia deliciosa leaf apoplast during biotrophic colonization by Pseudomonas syringae pv. actinidiae. J. Proteom. 101, 43-62. https://doi.org/10.1016/j. jprot.2014.01.030.

Petriccione, M., Mastrobuoni, F., Zampella, L., and Scortichini, M. (2015). Reference gene selection for normalization of RT-qPCR gene expression data from Actinidia deliciosa leaves infected with Pseudomonas syringae pv. actinidiae. Sci. Rep. 5, 16961. https://doi. org/10.1038/srep16961.

Reglinski, T., Vanneste, J., Wurms, K., Gould, E., Spinelli, F., and Rikkerink, E. (2013). Using fundamental knowledge of induced resistance to develop control strategies for bacterial canker of kiwifruit caused by Pseudomonas syringae pv. actinidiae. Front. Plant Sci. 4, 1-24. https://doi.org/10.3389/fpls.2013.00024.

Spinelli, F., Donati, I., Vanneste, J.L., Costa, M., and Costa, G. (2011). Real time monitoring of the interactions between Pseudomonas syringae pv. actinidiae and Actinidia species. Acta Hortic. 913, 461466. https://doi.org/10.17660/ActaHortic.2011.913.61.

Vanneste, J.L., Yu, J., Cornish, D.A., Tanner, D.J., Windner, R., Chapman, J.R., Taylor, R.K., Mackay, J.F., and Dowlut, S. (2013). Identification, virulence, and distribution of two biovars of Pseudomonas syringae pv. actinidiae in New Zealand. Plant Dis. 97, 708-719. https://doi. org/10.1094/PDIS-07-12-0700-RE.

Vanneste, J.L., Cornish, D.A., Yu, J., and Stokes, A. (2014). First report of Pseudomonas syringae pv. actinidiae the causal agent of bacterial canker of kiwifruit on Actinidia arguta vines in New Zealand. Plant Dis. 98, 418. https://doi.org/10.1094/PDIS-06-13-0667-PDN.

Weingart, H., Ullrich, H., Geider, K., and Volksch, B. (2001). The role of ethylene production in virulence of Pseudomonas syringae pvs. glycinea and phaseolicola, Phytopathol. 91, 511-518. https://doi. org/10.1094/PHYTO.2001.91.5.511.

Zheng, X., Spivey, N.W., Zeng, W., Liu, P., Fu, Z.Q., Klessig, D.F., He, S.Y., and Dong, X. (2012). Coronatine promotes Pseudomonas syringae virulence in plants by activating a signalling cascade that inhibits salicylic acid accumulation. Cell Host Microbe 11, 587-596. https:// doi.org/10.1016/j.chom.2012.04.014.

Received: Mar. 3, 2018

Accepted: Sep. 1, 2018 
Addresses of authors:

M. Nunes da Silva ${ }^{1,2}$, J. Machado ${ }^{2}$, G.M. Balestra ${ }^{3}$,

A. Mazzaglia ${ }^{3}$, M.W. Vasconcelos ${ }^{2}$ and S.M.P. Carvalho ${ }^{1, *}$

${ }^{1}$ GreenUPorto \& DGAOT, Faculdade de Ciências da

Universidade do Porto, Vairão, Portugal

${ }^{2}$ Universidade Católica Portuguesa, Centro de Biotecnologia

e Química Fina (CBQF), Laboratório Associado, Escola

Superior de Biotecnologia, Porto, Portugal

${ }^{3}$ Department of Agriculture and Forestry Science, Viterbo, Italy

* Corresponding author; E-mail: susana.carvalho@fc.up.pt

Tel.: (+351) 220402 000; Fax: (+351) 220402009 